\title{
Think STOP before going 'off-label'
}

\author{
Gerald Y Minuk MD FRCPC
}

$\mathrm{O}^{\prime \prime}$ nly after careful and extensive review of all available safety and efficacy data derived from preclinical and clinical trials will a regulatory body approve the licensing of a drug for a specific medical condition ('indication'). Experience with thalidomide, fialuridine and troglitazone, to name a few, reveal that despite these efforts, the process is not infallible. What then can we expect when a drug is used for a medical condition in which no such review process has been undertaken ('offlabel' use of the drug)?

Legally, physicians have the 'right' to prescribe any licensed drug for 'indicated' or 'off-label' use. Given that $88 \%$ of physicians have done so and $25 \%$ continue to do so on a daily basis, obviously physicians are exercising that right in large numbers (1). Elsewhere in this issue (pages 321-326), investigators at the University of Calgary suggest physicians consider using mycophenolate mofetil (MMF) for patients with autoimmune chronic hepatitis who fail to respond to or are intolerant of standard therapy (prednisone and/or azathioprine). Before implementing their suggestion, physicians may find it of value, if not prudent, to ask themselves the following STOP questions:

1. Side effects: what are the side effects of the agent being considered and can we expect a different side-effect profile when it is used 'off-label'?

2. Theoretical justification: do the properties of the drug fit with our understanding of the pathophysiology of the condition being treated?

3. Options: what other therapeutic options are available?

4. Patient-specific considerations: is this the right patient for this particular drug?

\section{Side effects}

MMF was extensively studied in the early 1990s as an immunosuppressive agent for use in patients undergoing organ transplantation. As a result, licensing trials were confined to transplant recipients and the side effects that were detected with its use were those identified in that specific patient population. However, for the most part and over the long term, organ recipients have normal or only mildly compromised hepatic function. The same cannot be said for patients with autoimmune chronic hepatitis who are either not receiving or are refractory to standard therapy. In these individuals, the liver disease is often active and, in many instances, quite advanced. Indeed, all five patients described in the Calgary report had stage 2 or higher fibrosis before MMF treatment. Given that MMF metabolism is impaired in the setting of advanced liver disease (2), one concern with 'off-label' use of MMF in this condition is a higher frequency and/or enhanced severity of side effects including myelosuppression (already a major concern in autoimmune chronic hepatitis patients who are predisposed to cytopenia due to their underlying immunological disorder and/or portal hypertension); opportunistic infections; diarrhea; and perhaps lymphoproliferative disease $(3,4)$. Unfortunately, the Calgary report contained too few patients to be able to determine whether this concern is valid.

\section{Theoretical justification}

Autoimmune chronic hepatitis is an immunologically mediated disease and MMF is a potent immunosuppressive agent. Thus, on the surface it seems reasonable to assume that MMF would be of value in patients with this condition. However, as the authors themselves point out, not all autoimmune disorders respond to all immunosuppressive agents. In the case of autoimmune chronic hepatitis, this concern is supported by the histological findings of the condition which consist of a predominantly plasma cell infiltration targeting hepatocytes, whereas in the transplant setting (where MMF is known to be of value), the cellular infiltration is largely lymphocytic and in the liver, focused on bile duct epithelial cells. Also of some concern regarding the efficacy of this agent are recent data from a national review of MMF in autoimmune chronic hepatitis, wherein response rates were clearly lower than those reported by the Calgary investigators (5).

\section{Options}

Before going 'off-label', the first option to be considered should always be a re-evaluation of standard care (eg, is the patient truly refractory to or intolerant of standard therapy?) Here, compliance is often an issue with patients either forgetting or 
choosing not to take their medications because of fears of reported side effects and/or their restored sense of well being. While pill counts may be of value, documentation of drug/metabolite levels or known physiological effects of treatment tend to be more informative (neither of which were described in the Calgary report). Another option for physicians that is often overlooked is not to treat. Not all patients with autoimmune chronic hepatitis progress to cirrhosis and liver failure. Indeed, in the patient population described by the Calgary investigators (mild to moderate serum aminotransferase abnormalities), the associated five- and 10-year life expectancies exceed $80 \%$ (6). Thus, not all patients warrant treatment, particularly when a drug is of uncertain value and time permits the identification of safe, effective and proven alternatives for that condition.

\section{Patient-specific features}

Patients are not homogenous. Some will be grateful for their physician's willingness to exhaust all therapeutic options on their behalf. This was obviously the intent of the Calgary investigators and for this they should be complimented. The medical community should also be grateful because these investigators have generated the essential preliminary data from which to base future, prospective, randomized, controlled clinical trials. However, other patients will take offense at such measures, considering themselves a 'guinea pig' in their physician's folly. Finally, there are some who will be inclined to resort to litigation (particularly if treatment goes awry). Although to date the courts have tended to support physicians, future 'not guilty' verdicts cannot be guaranteed $(7,8)$.
In addition, there is also the issue of cost. MMF is not inexpensive $(\$ 7,200 /$ year or six to seven times more expensive than azathioprine). In some individuals the additional cost will be borne by the province or third party payers; however, for others, the cost is theirs alone and may be prohibitive.

In summary, 'off-label' prescribing is a common and presumably often beneficial 'right' of physicians in clinical practice. However, on occasion, the side effects, theoretical justification, additional options and patient-specific features warrant forgoing that right, at least until the results of prospective, randomized controlled clinical trials are available.

\section{REFERENCES}

1. Serradell J, Galle B. Prescribing for unlabeled indications. HMO Pract 1993;7:44-7.

2. Parker G, Bullingham R, Kamm B, Hale M. Pharmacokinetics of oral mycophenolate mofetil in volunteer subjects with varying degrees of hepatic oxidative impairment. J Clin Pharmacol 1996;36:332-44.

3. Baudard M, Vincent M, Moreau P, Kergueris MF, Harousseau JL, Milpied N. Mycophenolate mofetil for the treatment of acute and chronic GVHD is effective and well tolerated but induces a high risk of infectious complications: a series of $21 \mathrm{BM}$ or PBSC transplant patients. Bone Marrow Transplant 2002;30:287-95.

4. Levy GA. Long-term immunosuppression and drug interactions. Liver Transpl 2001;7(Suppl 1):S53-9.

5. Chatur N, Ramji A, Bain VG, et al. Transplant immunosuppressive agents in non-transplant chronic autoimmune hepatitis: The CASL experience with mycophenolate mofetil and tacrolimus. Can J Gastroenterol 2004;18(Suppl A):94A. (Abst)

6. DeGroote J, Fevery J, Lepoutre L. Long-term follow-up of chronic active hepatitis of moderate severity. Gut 1978;19:510-3.

7. Blum RS. Legal considerations in off-label medication prescribing. Arch Intern Med 2002;162:1777-9.

8. Johnson LJ. Malpractice consult. Off-label prescribing and the standard of care. Med Econ 2001;78:97. 


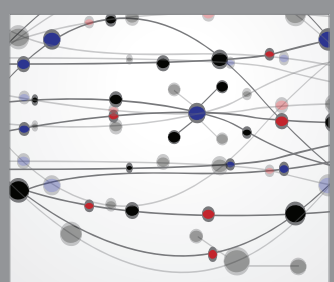

The Scientific World Journal
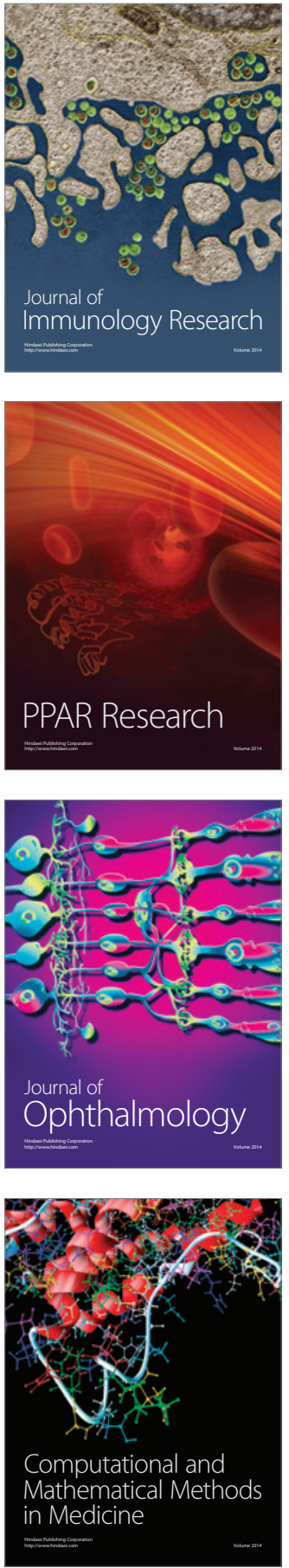

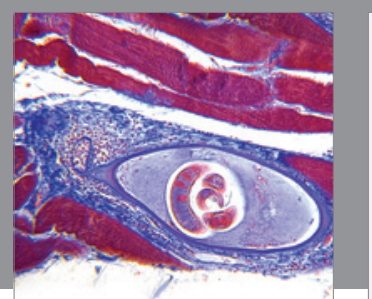

Gastroenterology Research and Practice

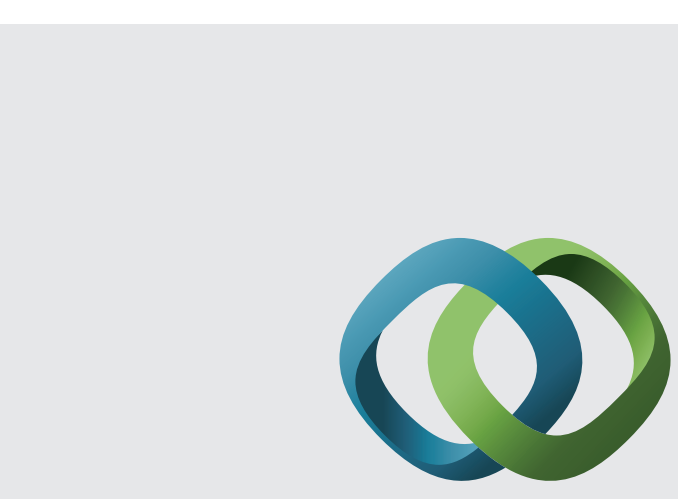

\section{Hindawi}

Submit your manuscripts at

http://www.hindawi.com
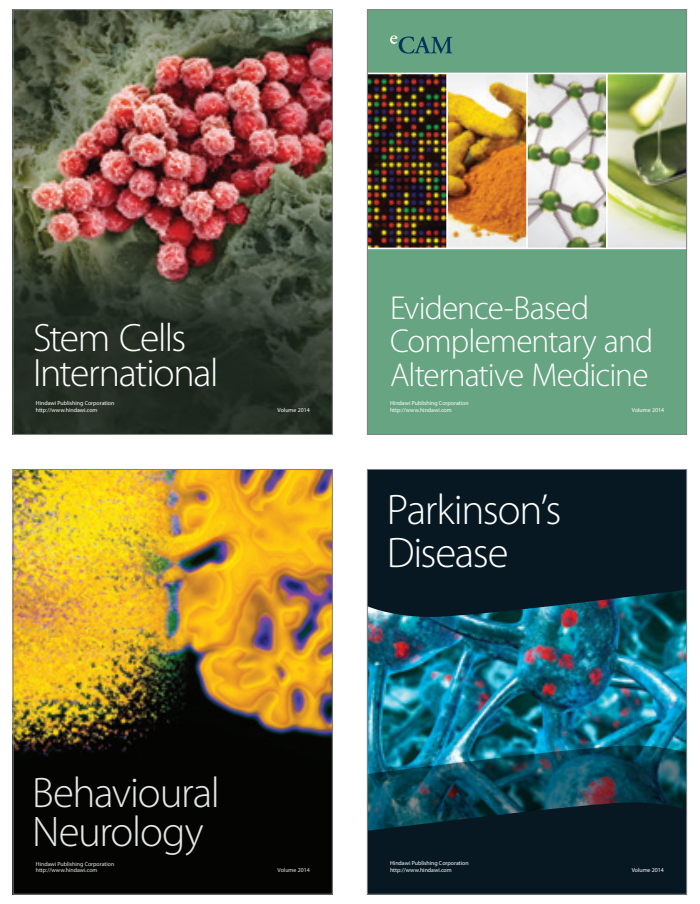
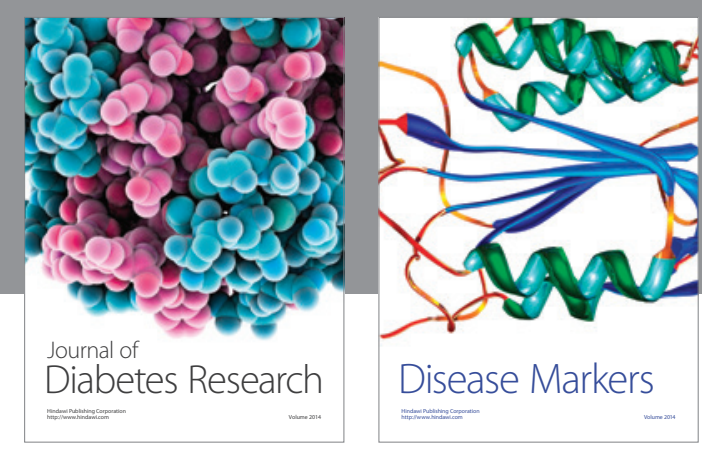

Disease Markers
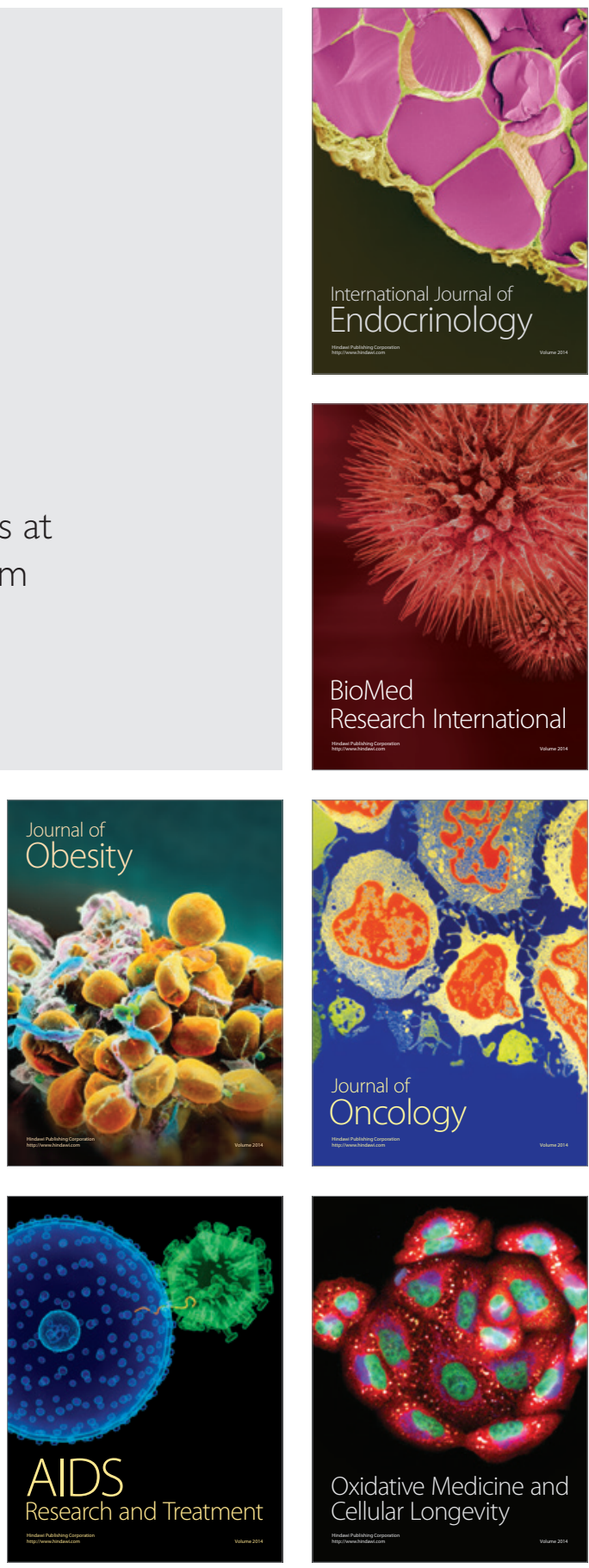